\title{
A Study on the Transmission Path of Zhang Daqian 's Paintings under the New Media Technology
}

\author{
Gao Dengming $^{1, a}$, Cao jingwei ${ }^{2, b}$
}

Zhang Daqian School of Fine Arts, Neijiang Normal University, Neijiang, 641100, China

aemail, bemail,

Keywords: Zhang Daqian 's Painting, New Media, Transmission Paths

\begin{abstract}
The present is in an age of "information explosion", and it's also a "reading and seeing pictures" or even a "listen to pictures" era. There are a lot of information every time in our lives.As the new media has the advantages of fast communication speed, strong interactivity, large information capacity and other advantages, it has become a new force in the information dissemination. Zhang Daqian 's painting ink splash of color, the combination of Chinese and western creative techniques and artistic effect heavy ink painting is the essence of Zhang Daqian's art. But in the new social environment, Zhang Daqian 's paintings are faced with several problems such as the single of transmission form, the small appreciation groups and other issues. In order to spread and develop Zhang Daqian 's art, make them to digital creation and editing, display the art of Zhang Daqian; Meanwhile,through the mobile phone apps, WeChats, micro-blogs, animations, videos and other new media paths, Zhang Daqian's art can not noly merely be combined with the times but also be spread to people's life
\end{abstract}

\section{Introduction}

Information fleeting in this new media age, many excellent artistic resources cannot be good spread due to the impact of a large number of living information, and some works are well treasured in the museums or the exhibition halls which makes them difficult to be appreciated by the public. Zhang Daqian 's paintings are also facing such a dilemma. These paintings provides us broad perspective and vigorous momentum, at the same time it has great artistic value in its drawing language. However, Zhang Daqian paintings in the new social environment, due to mostly paper works, resulting in its speed and scope of the transmission is limited, coupled with the current people's access to information, appreciation of the concept and living habits and differences in the past and other reasons, so Zhang Daqian Works to a certain extent, faced with the appreciation of small groups, a single form of performance, narrow range of applications and other issues. Therefore, Zhang Daqian art reasonable way of transmission is the focus of attention now.

\section{Zhang Daqian Art and Its Value}

Zhang Daqian was taught by Zeng Nongran when he was a child, then he showed his talent in painting in the flower of his age, afterwards he was called as East Zhang West Bi with Picasso. His works contain the essence of Eastern and Western art, in the Chinese ink painting and Western oil painting creation quite accomplished, he will be the ancient Chinese ink, ink painting techniques, and Western painting in the abstract, performance techniques integration. [1] In the traditional Chinese painting hook, dye, line and other traditional painting techniques, the ink directly spilled on the paper silk, let the ink with the paper silk flow, and then on the basis of ink splash, and finally with the hook, Point of the skills to complete the collection and shaping the work. His painting is not only a traditional Chinese painting a breakthrough, but also a breakthrough in the East and West culture and art, for the development of Chinese painting opened a new page, Zhang Daqian many works are wealth of art in China and all over the world [2] . 


\section{The Propagation Suffering of Zhang Daqian Art in the Digital Times}

Today, the society is well informationalized, and plenty of information is spread in digital way. In the new era background, the art works' value will be decreased if you can not quickly seize the initiative and get people's attention and attention. The excellent works come from the masses, and also need to go to the masses; they can lead the public, but above all need to serve people. Therefore, it's very important that finding and solve the issues in the transmission of Zhang Daqian Art.

Paper Type Paintings Communication Makes It Difficult For The Public To Easily Obtain. Nowadays the public are facing an unprecedented pressure, and everyone's life is just like the dominoes which they cannot control by themselves. Heavy works force the public make their ways in "steel frame" of their cities, meanwhile, staying home to read is really an extravagant hope. Internet +, cloud technology, and large data all the current popular terms. In the web 2.0 era, the data influence the people's way of life while also change the way of people getting information. The traditional culture is always mainly transmitted by newspapers, books, etc. For art, people can also gain the information from the museum, science and technology museum and other public cultural venues. But a few people will get information in the traditional way when people encounter the rapidly-developed network technology. Therefore, the new culture transmission and colorful forms of content are easier loved and accepted by public.

High Expenses of Artistic Works Make the Public Hard to Reach. Zhang Daqian was expert in whether landscape painting, flowers and birds painting, or freehand, meticulous, splash-ink and splash-color etc., especially in splashing. He took a very important position of painting industry in both China and abroad, called "South Zhang North Qi" with Qi Baishi, we can even say he made a merely deep influence. [3] All of these create the status and value of Zhang Daqian's painting art. For example, Zhang Daqian's splashing ink painting peach blossom spring created in 1982 was auctioned off on April 5, 2016 in Hong Kong Sotheby's spring auction with 240 million, and created a new record of Zhang Daqian's personal works auction record. [4] With the rapid development of contemporary economy, and the improving of people's living standards, spiritual life is also an important part that most people would like to pursue. In the art of highly market context, the diverse market demand makes the original opaque art prices more diverse. As a result, most of the best works of art in the high-end collectors, art museum wandering between, slowly reducing the exposure and the public missed. This is also anxious to prevent the spread and development of Zhang Daqian Art.

The Uneven Quality of Printing Influences the Spread of the Art Works Greatly. The transmission of paper is the most important way of transmitting Zhang Daqian's art. And what closely related to the paper transmission is printing, in a way, the effect of the art exhibition always have something to do with the quality of the copies. On the market, in view of the printing technology, distribution costs, shooting and scanning technology restrictions and other factors, ordinary paper prints exist low pixel, poor color and other issues. They were led to the common way of communication is difficult to achieve the good purpose of art communication, it is difficult to reflect the real charm of Zhang Daqian Art.

\section{The Analysis of the Digital Transmission Path of Zhang Daqian'S Art}

The development of digital technology directly determines the development of digital art, and also provides more possibilities for the realization of artistic aesthetic value. In the contemporary era, the popularization and application of electronic information technology has had a great impact on the existence of art, and the way of art production, storage, communication and acceptance, and even human thinking and perception is undergoing major changes. [5]

Zhang Daqian Art Combined with Cell Phone Intellective APP. Mobile phone as the main application of the current social tools, in the integration of each person's life but also to change the way people 's live. Especially in recent years, with the growing popularity of mobile phones, 
mobile terminal information transmission speed is faster and faster, they promoted the use and development of mobile phones. Now the mobile phone is not only the role of communication, but also a variety of functions, such as television, video games, newspapers, exhibitions, books and so on, the scope of the use of mobile phones is growing. According to the 21 Century Business Herald, report that minister of industry and information Miao Wei at the Communications Exhibition cum ICT China ----2016 high level forum opening speech said, until July this year,Chinese mobile phone users have a total of 1.304 billion, in a total of 4G users there are total of 646 million [6]. Therefore, the mobile phone user is a huge market, is to become one of the effective ways to achieve art popularization.Through the analysis of the spread of Zhang Daqian art on the mobile phone, put forward the appropriate communication program is the focus of attention. Mobile education APP development,should pay attention to young people's ideological education, healthy growth, cultural absorption, art training and other aspects of the problem. They can be in the wisdom of education, intelligent games, growth space and other aspects of the theme of the creation, combined with Zhang Daqian painting art in the phone App works of external and internal finish new change. Facing a huge mobile phone users,Zhang Daqian the spread of art not only needs the powerful media uninterrupted to display, also need art culture has its own advantages. Under the background of economic development, pay attention to the creation of culture and art, the promotion of cultural soft power, To achieve balanced development, transformation, connotation construction, effective coordination, to the more widespread of the art of painting for Mr Zhang Daqian to lay a solid foundation.

Zhang Daqian Art Combined with Micro-Blog and Wechat. New media technology based on digital technology, digital technology as the medium and carrier, so the new media is also known as digital new media [7].New media is the main camp Weibo and WeChat, because they are very importance cannot be ignored. Micro-blog and WeChat as the carrier for Zhang Daqian art communication,Zhang Daqian works of art, can be carried out in the form of high precision, high fidelity direct dissemination and display. With "online exhibition", "virtual interactive experience", "HD experience", "digital reappearance" in the network transmission. Doing so the way is no longer a simple photo in the emergence of the Internet, and turned into is a process to deal with the system to the related works of art on the Internet for highly accurate representation. First of all, using high precision scanner to scan. In order to eventually repeat produced and the original color of the same good products, all the required information must be scanned, obtained from the original. It can accurate scan the color of the original art works, hierarchy and three-dimensional sense of information, it can reduce the loss of the color, the level and the three-dimensional texture information caused by the original work by copying the two manuscript and scanning the original two times, it is an important index to improve the quality of reproduction. Secondly, adopt the three primary colors to give priority to process and color properties to represent painting color relations of objects. Art information collection is given priority to with three primary colors, short black version must be used for the skeleton of the process. Color version of the network order tuning range should be within the scope of the material printing eligibility as far as possible do long, black edition only skeleton role. In theory, is to give full play to network order limit and the limit of three primary colors ink density on the ground, reproduction of the rich texture of painting works and a strong dignified tone [8]. Thirdly, The use of high level of artistic treatment of painting works of digital art. To integrate the principles of color in the design and operation of image processing, handle color contrast principle, the principle of master-slave principle, the principle of space, the changes in temperature, etc., In each work's pursuit of unique temperament, style and color characteristics on the processing of light, achieve painting beauty, deep artistic conception, features strong artistic effect, as far as possible high precision again show works of art style and artistic characteristics. After complete a digital transformation of the work, will Zhang Daqian art digital resources according to the static, dynamic image, part of the image, the overall image, surrounding the image in the form of a complete data processing,to push through the related topics plates, increase the understanding of the people, finish transmission of Zhang Daqian art with highdefinition, rapid and wide range. 
Zhang Daqian Art Combined with Animation Art. Animation is an important part of China's cultural soft power. The animation industry in China in the early stage of development, produced a series of works including ink characteristics of experimental animation, such as "little tadpole looking for mom", "mountain and water", etc. But because of many factors, ink painting animation this work of art with Chinese characteristics, has not been good to inherit and carry forward. Today, our country has a good ink painting artists, have a industrialization production of animation company, there is no much more good color ink, black ink animation film. The combination of art and technology, art and culture industry innovation is the notable content. Add the painting effect and the skills of traditional Chinese painting in animation creation, use them formed related material background and content, by increasing the animation story content and visual effect, attract the attention of the audience, promote the development of culture creative art industry. At the same time, Zhang Daqian was born in Sichuan and he is very famous in the world. Zhang Daqian is also the Chinese unyielding, have the courage to fight. In the process of animation program research, according to the Zhang Daqian growth process, learning art, Zhang Daqian painting techniques such as the creation into animation small story, through accumulation of animation data, find new Zhang Daqian art ways. We should pay special attention to cultural undertakings creative work, the foundation of a new art form and the story content innovation, practical development, tell the Chinese story, promote the development of literature and art, show the Chinese new style.

\section{Conclusions}

New media and the Internet technology has changed the culture and art form, has given rise to a large number of new types of literature and art, but also brings the profound change of the literary ideas and practice of literature and art. In addition, digital, book visualization, reading the Internet changes, such as literary and social culture is faced with big changes. Therefore, in the face of the traditional culture and art, we need to adapt to the current situation, pays special attention to the production of literary and artistic creation. Meanwhile, in the face of Zhang Daqian painting art, we should make it more space, multiple paths to start a new transmission, promote the comprehensive development of Zhang Daqian Art. At the same time, we should promote Zhang Daqian Art into people's life, serving the public, flourishing literary, promote literary innovation.

\section{Acknowledgements}

The research work was supported by the following projects:

Sichuan Center for Zhang Daqian Studies, Project Number: ZDQ2017-11;

Neijiang Municipal Social Science Planning Project, Finance Number: Z017006;

Sichuan Provincial Department of Education Scientific Research Project, Project Number: 16SB0164;

National Training Programs of Innovation and Entrepreneurship for Undergraduates in Neijiang Normal University , project number: X201611

\section{References}

[1] Wan Yanyan. The artistic characteristics of Zhang Daqian ink splash color landscape painting [J]. stage.2015 (02)

[2] Liu Zheyi. Talk about Zhang Daqian's works with art history and artistic value [J]. in 2013 (21): 217-218.

[3] Wei Ruyu. Analysis Zhang Daqian's painting techniques about"the Yangtze River".2013 ([J].) (10) 
[4] Editorial department. Zhang Daqian "Taoyuan Painting" a new record of auction [J]. collector, 2016,05:90.

[5] Jin Hu. Ontology and aesthetic characteristics of digital art [J]. Journal of Hubei Institute of Fine Arts, 2015,01:82-84.

[6] Ling Zhen. Current situation and development strategy of domestic mobile phone marketing in China [J]. shopping mall modernization, 2015 (28): 37-38

[7] Tian Jinliang. On the influence of digital media on the spread of traditional culture [J]. art education research.2015 (23)

[8] Lian Wen. Oil painting reproduction and art micro jet [J]. printing technology, 2007, (10): 53-54. 